\title{
Distributed Switch-and-Stay Combining in Cognitive Relay Networks under Spectrum Sharing Constraints
}

\author{
Vo Nguyen Quoc Bao*, Trung Q. Duong ${ }^{\dagger}$, A. Nallanathan ${ }^{\ddagger}$, and George K. Karagiannidis $\S$ \\ * Posts and Telecommunications Institute of Technology, Vietnam. (e-mail: baovnq@ptithcm.edu.vn) \\ $\dagger$ Blekinge Institute of Technology, Sweden (e-mail: quang.trung.duong@bth.se)

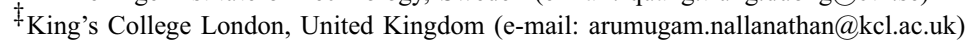 \\ $\S$ Aristotle University of Thessaloniki, 54124 Thessaloniki, Greece (e-mail: geokarag@auth.gr)
}

\begin{abstract}
Distributed switch-and-stay combining (DSSC) has been envisioned as an effective transmission technique to achieve spatial diversity in a distributed fashion, with low implementation complexity. In this paper, we take a step further to incorporate DSSC into spectrum-limited environment, where the operating nodes have to share the frequency radio spectrum with licensed users. In particular, by deploying DSSC scheme in cognitive radio networks, we have shown that the low transmit power at unlicensed users, inflicted by the peak interference power constraint at licensed users, can be alleviated. We present closedform expressions for outage probability and spectral efficiency, enabling us to evaluate and optimize the considered network performance. Numerical and simulation results show that when the switching threshold is below the outage threshold the full diversity order can be guaranteed at the secondary networks.
\end{abstract}

\section{INTRODUCTION}

Cognitive radio is getting more and more significant in future wireless systems, since the currently allocated radio spectrum is becoming more crowded as emphasized by the FCC [1]. Fortunately, the spectrum usage considerably varies in different time, frequency, and locations. As such, wireless terminals using cognitive radio are able to access the licensed band allocated to primary users (PUs) whenever PUs are quiescent, dramatically improving the spectrum utilization. In cognitive radio, secondary users (SUs) are allowed to opportunistically use the licensed band unless their transmission interferes with the normal communication of PUs in three modes: underlay, overlay, and interweave [2]. In the underlay mode, the most common scheme of cognitive radio, SUs are allowed to use the spectrum when the interference caused by SUs on PUs is within the range tolerated by PUs. In the overlay mode, SUs simultaneously share the same spectrum with PUs while improving or at least maintaining the transmission of PUs. In the interweave mode, SUs are only permitted to use the empty spectrum left by PUs. Among these three modes, the underlay mode appears to have many operational advantages and many research works have been devoted recently to study their network behaviors (see, e.g., [3], [4]). The main disadvantage of underlay approach is the limitation of transmit power for secondary transmitters to avoid any possible interference on PUs. As a potential solution, cooperative communications has been proposed recently for underlay cognitive networks to provide benefits of spatial diversity as compared to direct communications. More importantly, it is able to increase the secondary network coverage without imposing more interference on the primary networks. In [5], the authors derived the lower bound on the outage probability (OP) of selective decode-and-forward (DF) underlay relay networks by regarding the first hop signalto-noise ratio (SNR) as independent random variables. As such, the bound will become loose when the interference link is strong. The cooperative diversity gain in underlay cognitive relay systems over Rayleigh fading channels with three types of interference constraints was investigated in [6]. The numerical results showed that the full diversity order is achievable if the interference power constraint scales with the transmit power constraint. The best relay selection (BRS) for amplify-and-forward underlay networks was studied in [7] in terms of several performance metrics including OP, symbol error rate and ergodic capacity over Rayleigh fading channels.

Although the BRS has demonstrated its advantage in achieving the full spatial diversity gain [8]-[10], its relatively high complexity may hinder the practical implementation in some specific applications. Especially, it is not a trivial task to collect all the instantaneous channel state information (CSI) in the military or disaster sensor networks. As a result, as an alternative relaying, named distributed switch-and-stay combining (DSSC) scheme has been proposed in [11], where the relaying link can only be exploited as long as quality of the direct link is below a predefined quality-of-service (QoS). Since the DSSC scheme can achieve the full diversity gain as in BRS with lower complexity, it has gained great attention in the research literature for a non-cognitive scenario [12], [13].

To this end, in this paper, we first introduce the concept of DSSC for underlay cognitive networks in order to simplify the hardware complexity of cooperative communication (without using combining technique at the destination) as well as to improve the system spectral efficiency. The underlying idea is that rather than continually connecting the relaying link via the relay, the destination selects a particular link from either the relaying or the direct link for data transmission until its SNR quality drops below a given threshold. When this happens, the destination switches to another link and stays during the next time slot, regardless of whether or not the quality of that link is above or below the predetermined threshold. We then provide a new analytic framework to evaluate system performance metrics such as OP and spectral efficiency. It is important to note that for cognitive relay networks due to the appearance of a common random variable, i.e., the channel gain of the link 


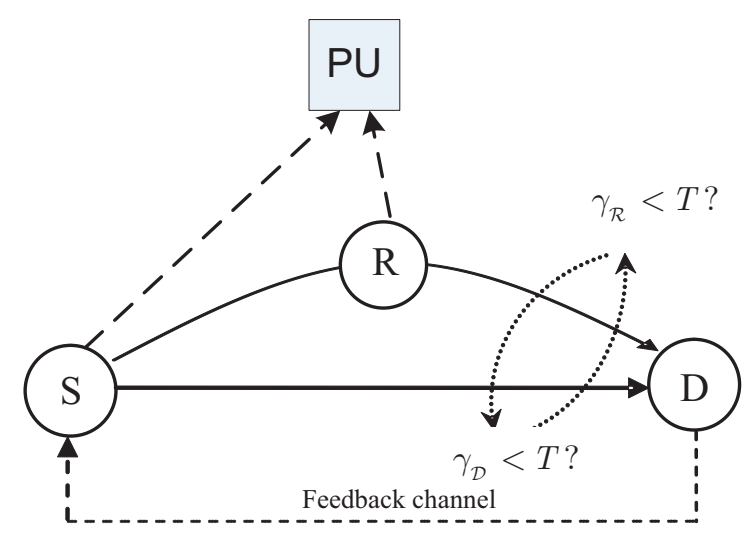

Fig. 1. Secondary underlay transmission with DSSC scheme.

from secondary transmitter to primary receiver, the analysis is related to the statistical dependence although the considered channels are independently distributed. To the best of our knowledge, this correlation effect has not been adequately addressed in the literature. As such, our analysis takes into account the correlation effect of cognitive DSSC networks, which is crucial to accurately evaluate system performance. In addition, the optimal problem of relay position with respect to the primary receiver position is also considered. The numerical and simulation results show that the system diversity and the optimal position of relay are functions of the switching threshold and the outage threshold, respectively. In particular, if the switching threshold is chosen lower than the outage threshold the system obtains full diversity gain. Otherwise, the diversity of one is attained.

\section{System ModeL}

We consider a DSSC transmission based cognitive radio network, referred to as secondary networks, operating in the coverage area of a primary network as shown in Fig. 1. The primary and the secondary networks share the same operating frequency band, which is licensed to the primary networks. The secondary network model is comprised of three nodes including a secondary source node $(\mathrm{S})$, a secondary destination node (D) and a relay node (R) coexisting with a primary node (P). Operating as per the rule of DSSC [11], the destination can receive data from the source either directly through the direct link or indirectly through the relaying link $(\mathrm{S} \rightarrow \mathrm{R} \rightarrow$ $\mathrm{D})$. In the case of receiving through the relaying link, each transmission period is divided in two sub-slots. In the first sub-slot, $\mathrm{S}$ transmits its data to the relay, the relay decodes the received signal and then forwards the re-encoded signals towards the destination in the second sub-slot. Assume that the destination is currently connected to the source via the $\mathrm{S} \rightarrow \mathrm{D}$ link (or $\mathrm{S} \rightarrow \mathrm{R} \rightarrow \mathrm{D}$ link). The destination will remain connected via the $\mathrm{S} \rightarrow \mathrm{D}$ link (or $\mathrm{S} \rightarrow \mathrm{R} \rightarrow \mathrm{D}$ link) as long as the instantaneous CSI of the $\mathrm{S} \rightarrow \mathrm{D}$ link (or $\mathrm{D} \rightarrow \mathrm{R} \rightarrow \mathrm{D}$ link) is above a given threshold, $T$. Otherwise, the destination will switch to the other link regardless of the instantaneous CSI on that link. To that effect, we assume that there exists an error-free feedback channel between the source and the destination and the switching will be effective in the next transmission slot. In practical systems, perfect CSI is not always available at the transmitter side, and the feedback CSI may be delayed or erroneous. However, it has been proved in [14] that the performance degradation is not considerable when the CSI error is smaller than the reciprocal of SNR. Furthermore, although the proposed scheme is based on uncoded system, it can be easily extended to coded systems.

As the PUs and SUs share the same frequency band under spectrum underlay manner, $\mathrm{SU}$ is allowed to simultaneously transmit with PUs as long as the communication of the PU is protected. Such simultaneous transmission results in interference signals observed at the primary receiver due to the existence of interference channels between the secondary transmitters (of the source and the relay) and the primary receiver. To represent the interference level at which the PU can still maintain reliable communication, we denote $I_{\mathrm{p}}$ as the maximum tolerable interference power of the primary receiver. As a result, the transmit powers for the secondary source and relay are constrained based on the CSI of the interference channels as

$$
P_{\mathrm{s}} \leq \frac{I_{\mathrm{p}}}{\left|h_{\mathrm{sp}}\right|^{2}}, P_{\mathrm{r}} \leq \frac{I_{\mathrm{p}}}{\left|h_{\mathrm{rp}}\right|^{2}},
$$

where $h_{\mathrm{sp}}$ and $h_{\mathrm{rp}}$ are the channel coefficients of the interference link from $\mathrm{S} \rightarrow \mathrm{P}$ and from $\mathrm{R} \rightarrow \mathrm{P}$, respectively. To enhance the network performance, we adopt the allowed maximum transmit power, i.e., $P_{\mathrm{s}}=\frac{I_{\mathrm{p}}}{\left|h_{\mathrm{sp}}\right|^{2}}$ for the source and $P_{\mathrm{r}}=\frac{I_{\mathrm{p}}}{\left|h_{\mathrm{rp}}\right|^{2}}$ for the relay. Here we assume that perfect CSI of each interference channel to the PU is available at the corresponding secondary transmitters. It can be done by, for example, approximating the overheard signals from the PR via the channel reciprocity property, being directly fed back from $\mathrm{PR}$, being provided from a band manger [15]. In this paper, we assume that the PU is far enough from SUs; thus we can model the primary inference to SUs as an equivalent additive Gaussian noise.

Moreover, we model all channels as independent complex Gaussian random variables leading to the quasi-static (slow) flat fading scenario. The complex valued channel gain between node $\mathrm{A} \in\{\mathrm{s}, \mathrm{r}\}$ and node $\mathrm{B} \in\{\mathrm{p}, \mathrm{r}, \mathrm{d}\}, h_{\mathrm{AB}}$, is thus distributed according to a Rayleigh fading distribution and the corresponding channel power, $\left|h_{\mathrm{AB}}\right|^{2}$, follows an exponential distribution with parameter $\lambda_{\mathrm{AB}}=\mathbb{E}\left\{\left|h_{\mathrm{AB}}\right|^{2}\right\}$.

Denoting $\gamma_{\mathcal{D}}, \gamma_{1}$ and $\gamma_{2}$ as the instantaneous SNR of the link from $\mathrm{S} \rightarrow \mathrm{D}, \mathrm{S} \rightarrow \mathrm{R}$ and $\mathrm{R} \rightarrow \mathrm{D}$, respectively, we have $\gamma_{\mathcal{D}}=\frac{I_{\mathrm{p}}}{N_{0}} \frac{\left|h_{\mathrm{sd}}\right|^{2}}{\left|h_{\mathrm{sp}}\right|^{2}}, \gamma_{1}=\frac{I_{\mathrm{p}}}{N_{0}} \frac{\left|h_{\mathrm{sr}}\right|^{2}}{\left|h_{\mathrm{sp}}\right|^{2}}$, and $\gamma_{2}=\frac{I_{\mathrm{p}}}{N_{\mathrm{o}}} \frac{\left|h_{\mathrm{rd}}\right|^{2}}{\left|h_{\mathrm{rp}}\right|^{2}}$. Under Rayleigh fading, the cumulative distribution function (CDF) 
of $\gamma_{i}$ with $i=\mathcal{D}, 1,2$ can be written as

$$
F_{\gamma_{i}}(\gamma)=\frac{\gamma}{\gamma+\alpha_{i}}
$$

where $\alpha_{0}=\frac{I_{\mathrm{p}}}{N_{0}} \frac{\lambda_{\mathrm{sd}}}{\lambda_{\mathrm{sp}}}, \alpha_{1}=\frac{I_{\mathrm{p}}}{N_{0}} \frac{\lambda_{\mathrm{sr}}}{\lambda_{\mathrm{sp}}}$, and $\alpha_{2}=\frac{I_{\mathrm{p}}}{N_{0}} \frac{\lambda_{\mathrm{rd}}}{\lambda_{\mathrm{rp}}}$. The corresponding probability density function (PDF) of $\gamma_{i}$ is written as

$$
f_{\gamma_{i}}(\gamma)=\frac{d F_{\gamma_{i}}(\gamma)}{d \gamma}=\frac{\alpha_{i}}{\left(\gamma+\alpha_{i}\right)^{2}}
$$

With the DF relaying, an outage occurs if either hop is in outage. Alternatively, it corresponds to the probability that the minimum SNR of the hops falls below the given outage threshold. Denoting $\gamma_{\mathcal{R}}$ as the end-to-end SNR of the dual DF relaying link, we can write

$$
\begin{aligned}
F_{\gamma_{\mathcal{R}}}(\gamma) & =\operatorname{Pr}\left[\left(\min \left(\gamma_{1}, \gamma_{2}\right) \leq \gamma\right]\right. \\
& =1-\operatorname{Pr}\left[\gamma_{1}>\gamma, \gamma_{2}>\gamma\right] \\
& =1-\frac{\alpha_{1} \alpha_{2}}{\left(\gamma+\alpha_{1}\right)\left(\gamma+\alpha_{2}\right)} .
\end{aligned}
$$

With the PDF and CDF of the $\gamma_{\mathcal{D}}$ and $\gamma_{\mathcal{R}}$ in hand, we are now able to study the system performance, which is given in detail in the next section.

\section{Outage Probability Analysis}

To analyze the system performance, we first establish the statistical relationship between the two links with the destination. Different from the conventional switch-and-stay combining (SSC), where dual-branches usually equally balance resulting in equality of average connecting time, the channels in DSSC are usually not identically distributed due to random distribution of relays leading to higher likelihood of the system staying in a "good" link and vice versa. To deal with this effect, we define the steady-stay selection probabilities for the two links as

$$
\begin{aligned}
& p_{\mathcal{D}}=p_{\mathcal{D}} \operatorname{Pr}\left(\gamma_{\mathcal{D}} \geq T\right)+p_{\mathcal{R}} \operatorname{Pr}\left(\gamma_{\mathcal{R}} \leq T\right), \\
& p_{\mathcal{R}}=p_{\mathcal{R}} \operatorname{Pr}\left(\gamma_{\mathcal{R}} \geq T\right)+p_{\mathcal{D}} \operatorname{Pr}\left(\gamma_{\mathcal{D}} \leq T\right),
\end{aligned}
$$

while we have

$$
p_{\mathcal{D}}+p_{\mathcal{R}}=1 .
$$

Solving (5a), (5b) and (6) yields

$$
\begin{aligned}
& p_{\mathcal{D}}=\frac{F_{\gamma_{\mathcal{R}}}(T)}{F_{\gamma_{\mathcal{D}}}(T)+F_{\gamma_{\mathcal{R}}}(T)}, \\
& p_{\mathcal{R}}=\frac{F_{\gamma_{\mathcal{D}}}(T)}{F_{\gamma_{\mathcal{D}}}(T)+F_{\gamma_{\mathcal{R}}}(T)} .
\end{aligned}
$$

The OP is an important performance metric, which is commonly used to assess the system performance. It is defined as the probability that the end-to-end SNR drops below a predefined threshold, $\gamma_{\text {th }}$. From the protocol description, two events, i.e. the currently connected link is below or greater the switching threshold, $T$, should be taken into account when calculating the system OP. In particular, when the SNR of the currently connected link is greater than the switching thresh- old, the system is in outage if and only if the instantaneous SNR is lower than the outage threshold. With the other case, i.e. the link switching is needed, the system will be in outage stage if the SNR of the other link drops below the outage threshold. However, due to the interference power constraint, these events, i.e. $\gamma_{\mathcal{D}}<T$ and $\gamma_{\mathcal{R}}<T$, are statistically dependent, which makes the conventional analysis method, for example [11], [12], inappropriate for the underlay spectrum sharing model.

Lemma 1: The OP of DSSC networks under underlay spectrum sharing can be expressed in closed-form as

$$
\mathrm{OP}=p_{\mathcal{D}}\left[\mathcal{A}\left(T, \gamma_{\mathrm{th}}\right)+\mathcal{B}(\mathcal{D})\right]+p_{\mathcal{R}}\left[\mathcal{A}\left(\gamma_{\mathrm{th}}, T\right)+\mathcal{B}(\mathcal{R})\right],
$$

where

$$
\mathcal{A}(x, y)=\frac{\frac{x}{\alpha_{0}}}{1+\frac{x}{\alpha_{0}}}-\frac{\alpha_{2}}{y+\alpha_{2}}\left[\frac{1}{1+\frac{y}{\alpha_{1}}}-\frac{1}{1+\frac{x}{\alpha_{0}}+\frac{y}{\alpha_{1}}}\right],
$$

with $x, y \in\left\{T, \gamma_{\text {th }}\right\}$ and

$$
\mathcal{B}(\mathrm{Z})=\left\{\begin{array}{cc}
0, & \gamma_{\mathrm{th}}<T \\
F_{\mathrm{Z}}\left(\gamma_{\mathrm{th}}\right)-F_{\mathrm{Z}}(T), & \gamma_{\mathrm{th}} \geq T
\end{array}\right.
$$

with $\mathrm{Z} \in\{\mathcal{D}, \mathcal{R}\}$

Proof: Grounded on the total probability theorem, the end-to-end OP is written as

$$
\begin{aligned}
\mathrm{OP}= & p_{\mathcal{D}}\left[\operatorname{Pr}\left(\gamma_{\mathcal{D}} \leq T\right) \operatorname{Pr}\left(\gamma_{\mathcal{R}} \leq \gamma_{\mathrm{th}} \mid \gamma_{\mathcal{D}} \leq T\right)\right. \\
& \left.+\operatorname{Pr}\left(\gamma_{\mathcal{D}}>T\right) \operatorname{Pr}\left(\gamma_{\mathcal{D}} \leq \gamma_{\mathrm{th}} \mid \gamma_{\mathcal{D}}>T\right)\right] \\
+ & p_{\mathcal{R}}\left[\operatorname{Pr}\left(\gamma_{\mathcal{R}} \leq T\right) \operatorname{Pr}\left(\gamma_{\mathcal{D}} \leq \gamma_{\mathrm{th}} \mid \gamma_{\mathcal{R}} \leq T\right)\right. \\
& \left.+\operatorname{Pr}\left(\gamma_{\mathcal{R}}>T\right) \operatorname{Pr}\left(\gamma_{\mathcal{R}} \leq \gamma_{\mathrm{th}} \mid \gamma_{\mathcal{R}}>T\right)\right]
\end{aligned}
$$

Using the conditional probability, (10) can be simplified as

$$
\begin{aligned}
\mathrm{OP} & =p_{\mathcal{D}}\left[\operatorname{Pr}\left(\gamma_{\mathcal{D}} \leq T, \gamma_{\mathcal{R}} \leq \gamma_{\mathrm{th}}\right)+\operatorname{Pr}\left(\gamma_{\mathcal{D}} \leq \gamma_{\mathrm{th}}, \gamma_{\mathcal{D}}>T\right)\right] \\
& +p_{\mathcal{R}}\left[\operatorname{Pr}\left(\gamma_{\mathcal{R}} \leq T, \gamma_{D} \leq \gamma_{\mathrm{th}}\right)+\operatorname{Pr}\left(\gamma_{\mathcal{R}} \leq \gamma_{\mathrm{th}}, \gamma_{\mathcal{R}}>T\right]\right.
\end{aligned}
$$

It is straightforward to recognize that $\gamma_{\mathcal{D}}$ and $\gamma_{\mathcal{R}}$ are correlated due to the common term, $\left|h_{\mathrm{sp}}\right|^{2}$, resulting in

$$
\operatorname{Pr}\left(\gamma_{\mathcal{D}} \leq T, \gamma_{\mathcal{R}} \leq \gamma_{\mathrm{th}}\right) \neq \operatorname{Pr}\left(\gamma_{\mathcal{D}} \leq T\right) \operatorname{Pr}\left(\gamma_{\mathcal{R}} \leq \gamma_{\mathrm{th}}\right)
$$

Furthermore, we can see that the two terms in (11), i.e., $\operatorname{Pr}\left(\gamma_{\mathcal{D}} \leq T, \gamma_{\mathcal{R}} \leq \gamma_{\text {th }}\right)$ and $\operatorname{Pr}\left(\gamma_{\mathcal{R}} \leq T, \gamma_{\mathcal{D}} \leq \gamma_{\text {th }}\right)$, are taken from the similar form as $\mathcal{A}(x, y)=\operatorname{Pr}\left(\gamma_{\mathcal{D}} \leq x, \gamma_{\mathcal{R}} \leq y\right)$ with $x, y \in\left\{T, \gamma_{\mathrm{th}}\right\}$. Thanks to the statistical independence among channels, it is possible to evaluate $\mathcal{A}(x, y)$,

$$
\mathcal{A}(x, y)=\mathbb{E}_{\gamma_{I}}\left\{\operatorname{Pr}\left(\gamma_{\mathcal{D}} \leq x \mid \gamma_{I}\right) \operatorname{Pr}\left(\gamma_{\mathcal{R}} \leq y \mid \gamma_{I}\right)\right\},
$$

where $\gamma_{I}=\left|h_{\mathrm{sp}}\right|^{2}$. Rewriting (13) under an explicit form, we have

$$
\mathcal{A}(x, y)=\int_{0}^{\infty} \operatorname{Pr}\left(\gamma_{\mathcal{D}} \leq x \mid \gamma_{I}\right) \operatorname{Pr}\left(\gamma_{\mathcal{R}} \leq y \mid \gamma_{I}\right) f_{\gamma_{I}}\left(\gamma_{I}\right) d \gamma_{I},
$$


where $\operatorname{Pr}\left(\gamma_{\mathcal{D}} \leq x \mid \gamma_{I}\right)$ is derived as follows:

$$
\operatorname{Pr}\left(\gamma_{\mathcal{D}} \leq x \mid \gamma_{I}\right)=1-\exp \left(-\frac{x \gamma_{I}}{\frac{I_{\mathrm{p}}}{N_{0}} \lambda_{0}}\right) .
$$

For the relaying link, we rewrite $\operatorname{Pr}\left(\gamma_{R} \leq y \mid \gamma_{I}\right)$ as

$$
\begin{aligned}
\operatorname{Pr}\left(\gamma_{\mathcal{R}} \leq y \mid \gamma_{I}\right) & =\operatorname{Pr}\left[\min \left(\gamma_{1}\left|\gamma_{I}, \gamma_{2}\right| \gamma_{I}\right)<y\right] \\
& =1-\operatorname{Pr}\left(\gamma_{1}>y \mid \gamma_{I}\right) \operatorname{Pr}\left(\gamma_{2}>y \mid \gamma_{I}\right) .
\end{aligned}
$$

Making use of the fact that $\operatorname{Pr}\left(\gamma_{2}>y \mid \gamma_{I}\right)=\operatorname{Pr}\left(\gamma_{2}>y\right)$, we have

$$
\operatorname{Pr}\left(\gamma_{\mathcal{R}} \leq y \mid \gamma_{I}\right)=1-\exp \left(-\frac{y \gamma_{I}}{\frac{I_{\mathrm{p}}}{N_{0}} \lambda_{1}}\right) \frac{\alpha_{2}}{y+\alpha_{2}} .
$$

Substituting (16) and (17) back into the expression of (14) and then performing the integration, followed by some manipulations, we have

$$
\begin{aligned}
\mathcal{A}(x, y)= & \int_{0}^{\infty}\left[1-\exp \left(-\frac{x \gamma_{I}}{\frac{I_{\mathrm{p}}}{N_{0}} \lambda_{0}}\right)\right] \\
& \times\left[1-\exp \left(-\frac{y \gamma_{I}}{\frac{I_{\mathrm{p}}}{N_{0}} \lambda_{1}}\right) \frac{\alpha_{2}}{y+\alpha_{2}}\right] \frac{1}{\lambda_{I}} e^{-\frac{\gamma_{I}}{\lambda_{I}}} d \gamma_{I} \\
= & \frac{\frac{x}{\alpha_{0}}}{1+\frac{x}{\alpha_{0}}}-\frac{\alpha_{2}}{y+\alpha_{2}}\left(\frac{1}{1+\frac{y}{\alpha_{1}}}-\frac{1}{1+\frac{x}{\alpha_{0}}+\frac{y}{\alpha_{1}}}\right)
\end{aligned}
$$

where $\lambda_{I}=\mathbb{E}\left\{\gamma_{I}\right\}$. Now we calculate the conditioned OPs, $\operatorname{Pr}\left(\gamma_{\mathcal{D}} \leq \gamma_{\mathrm{th}}, \gamma_{\mathcal{D}}>T\right)$ and $\operatorname{Pr}\left(\gamma_{\mathcal{R}} \leq \gamma_{\mathrm{th}}, \gamma_{\mathcal{R}}>T\right)$ in (11), which are taken from the general form

$$
\begin{aligned}
\mathcal{B}(\mathrm{Z}) & =\operatorname{Pr}\left(\gamma_{\mathbf{Z}} \leq \gamma_{\mathrm{th}}, \gamma_{\mathbf{Z}}>T\right) \\
& =\left\{\begin{array}{cl}
0, & T>\gamma_{\mathrm{th}} \\
F_{\gamma_{\mathbf{Z}}}\left(\gamma_{\mathrm{th}}\right)-F_{\gamma_{\mathbf{Z}}}(T), & T \leq \gamma_{\mathrm{th}}
\end{array}\right.
\end{aligned}
$$

with $Z \in\{\mathcal{D}, \mathcal{R}\}$. Finally, putting everything together, i.e., (18), (19) and (11), we have the end-to-end OP for DSSC networks under interference constraints as (9).

Lemma 2: The OP of DSSC networks under underlay spectrum sharing can be asymptotically approximated as

$$
\mathrm{OP} \approx\left\{\begin{array}{cl}
\frac{T}{\alpha_{0}}\left(\frac{2 \gamma_{\mathrm{th}}}{\alpha_{1}}+\frac{\gamma_{\mathrm{th}}}{\alpha_{2}}\right) & , T>\gamma_{\mathrm{th}} \\
\frac{2\left(\gamma_{\mathrm{th}}-T\right)}{1+\frac{\lambda_{\mathrm{sd}}}{\lambda_{\mathrm{sr}}}+\frac{\lambda_{\mathrm{sd}} \lambda_{\mathrm{rp}}}{\lambda_{\mathrm{sp}} \lambda_{\mathrm{rd}}}}\left(\frac{1}{\alpha_{1}}+\frac{1}{\alpha_{2}}\right) & , T \leq \gamma_{\mathrm{th}}
\end{array}\right.
$$

Proof: We start the proof by considering $p_{\mathcal{R}}$ and $p_{\mathcal{D}}$. In particular, we re-write $p_{\mathcal{R}}$ as

$$
p_{\mathcal{R}}=\frac{1}{1+\frac{F_{\gamma_{\mathcal{R}}}(T)}{F_{\gamma_{\mathcal{D}}}(T)}} .
$$

Using McLaurin series expansion for (21) and then neglecting higher-order terms gives

$$
p_{\mathcal{R}} \approx \frac{1}{1+\frac{\alpha_{0}}{\alpha_{1}}+\frac{\alpha_{0}}{\alpha_{2}}} .
$$

Recalling that $p_{\mathcal{R}}+p_{\mathcal{D}}=1$, we immediately arrive at

$$
p_{\mathcal{D}}=1-p_{\mathcal{R}} \approx \frac{\frac{\alpha_{0}}{\alpha_{1}}+\frac{\alpha_{0}}{\alpha_{2}}}{1+\frac{\alpha_{0}}{\alpha_{1}}+\frac{\alpha_{0}}{\alpha_{2}}} .
$$

We are now in a position to approximate $\mathcal{A}(x, y)$ in the high SNR regime. Following the same approach as for $p_{\mathcal{R}}$, we have

$$
\mathcal{A}(x, y) \approx \frac{x y}{\alpha_{0}}\left[\frac{2}{\alpha_{1}}+\frac{1}{\alpha_{2}}\right] .
$$

Similarly, $\operatorname{Pr}\left(\gamma_{\mathcal{D}} \leq \gamma_{\mathrm{th}}, \gamma_{\mathcal{D}}>T\right)$ and $\operatorname{Pr}\left(\gamma_{\mathcal{R}} \leq \gamma_{\mathrm{th}}, \gamma_{\mathcal{R}}>T\right)$ for the case $T<\gamma_{\mathrm{th}}$ can be approximated, respectively, as follows:

$$
\begin{aligned}
& \operatorname{Pr}\left(\gamma_{\mathcal{D}} \leq \gamma_{\mathrm{th}}, \gamma_{\mathcal{D}}>T\right)=F_{\gamma_{\mathcal{D}}}\left(\gamma_{\mathrm{th}}\right)-F_{\gamma_{\mathcal{D}}}(T) \\
& =\frac{\gamma_{\mathrm{th}}}{\gamma_{\mathrm{th}}+\alpha_{0}}-\frac{T}{T+\alpha_{0}} \approx \frac{\gamma_{\mathrm{th}}-T}{\alpha_{0}},
\end{aligned}
$$

and

$$
\begin{aligned}
& \operatorname{Pr}\left(\gamma_{\mathcal{R}} \leq \gamma_{\mathrm{th}}, \gamma_{\mathcal{R}}>T\right)=F_{\gamma_{\mathcal{R}}}\left(\gamma_{\mathrm{th}}\right)-F_{\gamma_{\mathcal{R}}}(T) \\
& =\frac{1}{\left(1+\frac{T}{\alpha_{1}}\right)\left(1+\frac{T}{\alpha_{2}}\right)}-\frac{1}{\left(1+\frac{\gamma_{\mathrm{th}}}{\alpha_{1}}\right)\left(1+\frac{\gamma_{\mathrm{th}}}{\alpha_{2}}\right)} \\
& \approx\left(\gamma_{\mathrm{th}}-T\right)\left(\frac{1}{\alpha_{1}}+\frac{1}{\alpha_{2}}\right) .
\end{aligned}
$$

Finally, combining (21), (23), (24), (25) and (26), we can obtain the desired result, as shown in (20), which also completes the proof.

Lemma 3: Cognitive relay networks achieve the full diversity order of two if and only if the switching threshold $(T)$ is greater than or equal to the SNR threshold $\left(\gamma_{\mathrm{th}}\right)$. Otherwise, only diversity order of one is attained.

Proof: The proof is straightforward by inferring from (20).

Lemma 4: At high SNRs, the OP achieves its minimum given by

$$
\frac{T^{2}}{\alpha_{0}}\left(\frac{2}{\alpha_{1}}+\frac{1}{\alpha_{2}}\right)
$$

if and only if the switching threshold is equal to the outage threshold, i.e., $\gamma_{\mathrm{th}}=T$.

Proof: From (20), it is easy to observe that for a given $\gamma_{\text {th }}$, OP will increase if $T$ increases. As a result, the OP is minimum at $\gamma_{\mathrm{th}}=T$. It should be noted that for $\gamma_{\mathrm{th}}>T$ the OP is greater than that of $\gamma_{\mathrm{th}}=T$ (see Lemma 2).

\section{Spectral EfFiciency}

The system spectral efficiency is a useful and insightful metric, which is defined as

$$
\mathrm{ASE}=p_{\mathcal{D}} r+p_{\mathcal{R}} \frac{r}{2},
$$

where $r$ represents the average spectral efficiency of direct transmission. Plugging (5a) and (5b) into (28), one has

$$
\mathrm{ASE}=r\left[1-\frac{\frac{1}{2}}{1+\left(\frac{T+\alpha_{0}}{T}\right)\left(1-\frac{\alpha_{1} \alpha_{2}}{\left(T+\alpha_{1}\right)\left(T+\alpha_{2}\right)}\right)}\right] .
$$


Furthermore, considering (25) and (26), the system spectral efficiency is approximated as

$$
\mathrm{ASE} \rightarrow r\left(1-\frac{1 / 2}{1+\frac{\alpha_{0}}{\alpha_{1}}+\frac{\alpha_{0}}{\alpha_{2}}}\right)
$$

\section{Optimal Relay Replacement}

In this section, we find an optimal relay placement scheme for the proposed protocol, which is able to minimize the system OP. Since the exact form of OP is not trivial, we present here the approximate optimal solution based on (20). Simulation results later will show that the approximate optimal solution is valid and outperforms the randomized relay replacement. For simplicity, we consider 2-D linear networks where the secondary relay is placed on a straight line connecting the secondary source and the secondary destination, whose distance is normalized to one. Denoting $d$ as the distance from the secondary source to the secondary relay, the coordinates of the secondary source, relay and destination are respectively given as $(0,0),(d, 0)$, and $(1,0)$.

Theorem 1: For the given coordinate of the primary receiver $\left(x_{\mathrm{P}}, y_{\mathrm{P}}\right)$, the system OP will achieve its minimum if and only if the relay is placed at

$$
d^{*}=-\frac{x_{\mathrm{P}}}{2}-\frac{1}{2} \sqrt{\Phi_{0}+\Phi_{4}}+\frac{1}{2} \sqrt{2 \Phi_{0}-\Phi_{4}-\frac{\Phi_{3}}{2 \Phi_{0}+\Phi_{4}}},
$$

where

$$
\begin{aligned}
\Phi_{0}= & x_{\mathrm{P}}^{2}-\frac{2}{3}(1-\kappa) d_{\mathrm{sp}}^{2}, \\
\Phi_{1}= & d_{\mathrm{sp}}^{4}(1-\kappa)^{2}+12 d_{\mathrm{sp}}^{2} \kappa\left(\mathrm{x}_{\mathrm{P}}-1\right), \\
\Phi_{2}= & 2 d_{\mathrm{sp}}^{4}(1-\kappa)^{2}+108 d_{\mathrm{sp}}^{4} \kappa^{2}-108 d_{\mathrm{sp}}^{2} \mathrm{x}_{\mathrm{P}}^{2} \kappa \\
& +27 d_{\mathrm{sp}}^{4}(1-\kappa) \kappa+36 d_{\mathrm{sp}}^{4} \mathrm{x}_{\mathrm{P}}(1-\kappa) \kappa \\
\Phi_{3}= & 8 x_{\mathrm{P}}^{3}-8 d_{\mathrm{sp}}^{4} x_{\mathrm{P}}(1-\kappa)-16 d_{\mathrm{sp}}^{4} \kappa, \\
\Phi_{4}= & \frac{\Phi_{1}}{3} \sqrt[3]{\frac{2}{\Phi_{2}+\sqrt{\Phi_{2}-4 \Phi_{1}}}}+\frac{1}{3} \sqrt[3]{\frac{\Phi_{2}+\sqrt{\Phi_{2}-4 \Phi_{1}}}{2}},
\end{aligned}
$$

and

$$
d_{\mathrm{sp}}=\sqrt{x_{\mathrm{P}}^{2}+y_{\mathrm{P}}^{2}}, \quad \kappa=\left\{\begin{array}{ll}
2^{\frac{2}{\eta}}, & T \geq \gamma_{\mathrm{th}} \\
1, & T<\gamma_{\mathrm{th}}
\end{array} .\right.
$$

Proof: From (20), we can see two distinct cases to consider separately, that is $T \geq \gamma_{\mathrm{th}}$ and $T<\gamma_{\mathrm{th}}$. For the second case, minimizing the objective function is equivalent to minimizing $\frac{1}{\alpha_{1}}+\frac{1}{\alpha_{2}}$. As a result, the optimization problem for both cases can be unified as

$$
\min \frac{\zeta}{\alpha_{1}}+\frac{1}{\alpha_{2}}
$$

where $\zeta=\left\{\begin{array}{ll}2, & T \geq \gamma_{\mathrm{th}} \\ 1, & T<\gamma_{\mathrm{th}}\end{array}\right.$. Since $\alpha_{1}$ and $\alpha_{2}$ are all positive, applying the Cauchy-Schwartz inequality, we have

$$
\frac{\zeta}{\alpha_{1}}+\frac{1}{\alpha_{2}} \geq 2 \sqrt{\frac{\zeta}{\alpha_{1} \alpha_{2}}} \text {. }
$$

Equality occurs if and only if $\alpha_{1}=\zeta \alpha_{2}$. Taking into account the pathloss effect, i.e., $\lambda_{\mathrm{AB}}=d_{\mathrm{AB}}{ }^{-\eta}$, where $\eta$ denotes the path loss exponent, we have

$$
\frac{d_{\mathrm{sp}}}{d}=\zeta^{\frac{1}{\eta}} \frac{d_{\mathrm{rp}}}{1-d}
$$

where $d_{\mathrm{sp}}=\left(x_{\mathrm{P}}^{2}+y_{\mathrm{P}}^{2}\right)^{1 / 2}$. As per the law of cosines, we can write

$$
d_{\mathrm{rp}}^{2}=d^{2}+d_{\mathrm{sp}}^{2}-2 d x_{\mathrm{P}} .
$$

Combining (35) and (36) yields a quadratic form of unknown $d$ as follows:

$$
d^{4}-2 x_{\mathrm{P}} d^{3}+{d_{\mathrm{sp}}}^{2}(1-\kappa) d^{2}+2 \kappa d_{\mathrm{sp}}^{2} d-\kappa d_{\mathrm{sp}}^{2}=0,
$$

where $\kappa=\left\{\begin{array}{ll}2^{\frac{2}{\eta}}, & T \geq \gamma_{\mathrm{th}} \\ 1, & T<\gamma_{\mathrm{th}}\end{array}\right.$. Denote $\Psi(d)=d^{4}-2 x_{\mathrm{P}} d^{3}+$ $d_{\mathrm{sp}}(1-\kappa) d^{2}+2 \kappa d_{\mathrm{sp}}^{2} d-\kappa d_{\mathrm{sp}}^{2}$, we have $\Psi(0)=-d_{\mathrm{sp}}{ }^{2}<0$ and $\Psi(1)=\left(1-x_{\mathrm{P}}\right)^{2}+y_{\mathrm{P}}^{2}>0$ resulting in $\Psi(0) \Psi(1)<0$. It suffices to state that the optimal relay position always exists for the proposed networks. The optimal value of $d$, denoted as $d^{*}$, is one of the roots of the above quadratic equation and satisfies the constraint of $0 \leq d^{*} \leq 1$ as shown (31).

\section{NUMERICAL RESULTS}

In this section, we present simulation performance results to verify the preceding analysis and to study the system behaviours. The channel and system settings are chosen as follows: $d=0.5$ and $\eta=3$. The primary receiver is located at coordinates $\left(x_{\mathrm{P}}, y_{\mathrm{P}}\right)=(0.5,0.5)$.

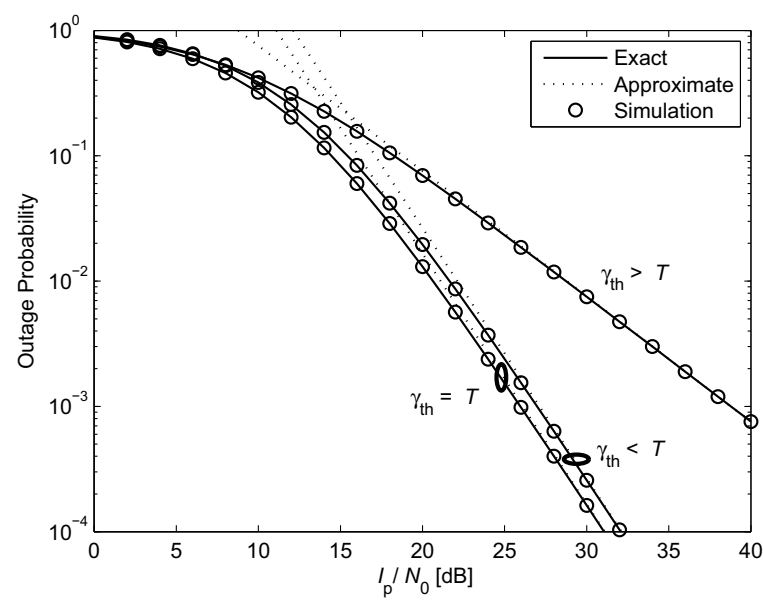

Fig. 2. Outage probability versus average SNRs.

In Fig. 2, we plot the OP versus average SNRs for the three different cases of $T$ and $\gamma_{\text {th }}$ with $\gamma_{\text {th }}=5$ and $T \in\{2,5,8\}$, i.e., Case 1: $\gamma_{\mathrm{th}}=T$, Case 2: $\gamma_{\mathrm{th}}<T$, and Case 3: $\gamma_{\mathrm{th}}>T$. This figure shows that the simulation results match perfectly with analytical results. The first two cases provide the full diversity gain while the last case has only diversity gain of one. Among them, Case 1 provides the best performance. It 
is also straightforwardly observed that the gap between curves of the first two cases is $10 \log _{10}\left(T-\gamma_{\text {th }}\right) \mathrm{dB}$. Note that this observation is the same as the conventional SSC network and the DSSC network without cognitive radio.

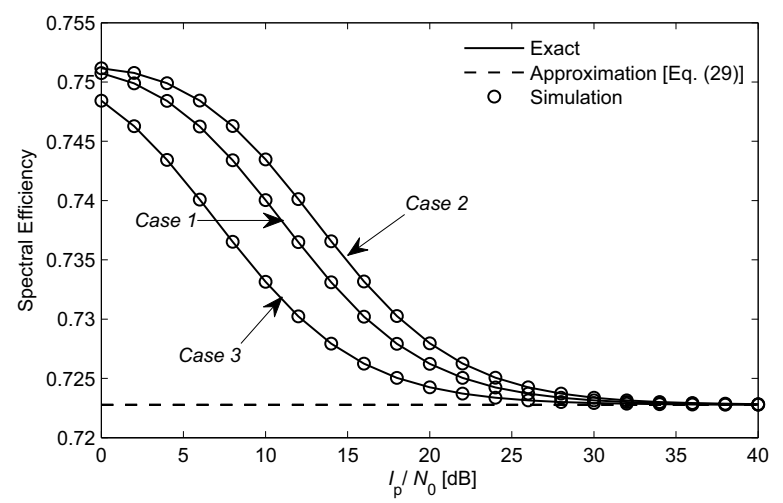

Fig. 3. Spectral efficiency versus average SNRs.

In Fig. 3, the system spectral efficiency versus average SNRs corresponding to the three cases are shown. Different from what we can see in Fig. 2, Case 3 has the best system spectral efficiency and Case 2 is the worst. It is worthy noting that choosing $\gamma_{\text {th }}=T$ will provide full diversity and better spectral efficiency as compared to the case of choosing $\gamma_{\mathrm{th}}>T$.

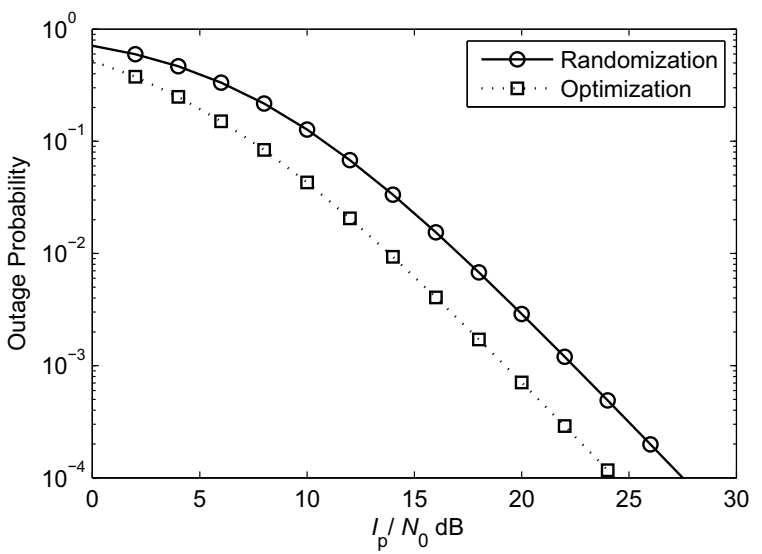

Fig. 4. Outage probability versus average SNRs.

In Fig. 4, we study performance advantage offered by the proposed optimal relay replacement (denoted as PROFILE A). As a baseline, we also consider the natural relay replacement profile denoted by ProfiLe B. In ProfiLE B, the x-coordinate is chosen randomly from a uniform distribution. As expected, Profile A outperforms PROFILE B. In particular, we can gain around $4 \mathrm{~dB}$ since the relay position is optimized instead of randomly chosen. It is due to the fact that in underlay cooperative networks, the optimal relay position depends not only on the average channel gain of data links but also on the average channel gain of interference links.

\section{CONCLUSIONS}

In this paper, we investigated the performance of DSSC scheme in cognitive radio networks by deriving the closedform expressions for $\mathrm{OP}$ and spectral efficiency. We also obtained the optimal relay location with respect to the position of primary receiver. Moreover, the mutual relationship between the switching threshold of DSSC and outage threshold of the considered system has been considered. This important finding reveals the fact that when the switching threshold is less than the outage threshold the DSSC scheme exhibits the full diversity order under spectrum-sharing environment and vice versa.

\section{ACKNOWLEDGMENT}

This research was supported by the Vietnam's National Foundation for Science and Technology Development (NAFOSTED) (No. 102.04-2012.20).

\section{REFERENCES}

[1] FCC, "Spectrum policy task force report," ET Docket 02-155, no. 11, 2002.

[2] A. Goldsmith, S. A. Jafar, I. Maric, and S. Srinivasa, "Breaking spectrum gridlock with cognitive radios: An information theoretic perspective," Proc. IEEE, vol. 97, no. 5, pp. 894-914, May 2009.

[3] H. Guo and V. Leung, "Orthogonal transmissions for spectrum underlay miso cognitive radio," IEEE Trans. Wireless Commun., vol. 11, no. 4 pp. 1266-1270, Apr. 2012.

[4] J. pyo Hong, H. Bi, B. Tae Won, and C. Wan, "On the cooperative diversity gain in underlay cognitive radio systems," IEEE Trans. Commun., vol. 60, no. 1, pp. 209-219, Jan. 2012.

[5] L. Luo, P. Zhang, G. Zhang, and J. Qin, "Outage performance for cognitive relay networks with underlay spectrum sharing," IEEE Commun. Lett., vol. 15, no. 7, pp. 710-712, Jul. 2011.

[6] S. Sagong, J. Lee, and D. Hong, "Capacity of reactive DF scheme in cognitive relay networks," IEEE Trans. Wireless Commun., vol. 10, no. 10 , pp. 3133-3138, Oct. 2011.

[7] V. N. Q. Bao, T. Q. Duong, D. Da Costa, G. Alexandropoulos, and A. Nallanathan, "Cognitive amplify-and-forward relaying with best relay selection in non-identical Rayleigh fading," IEEE Commun. Lett., vol. 17, no. 3, pp. 475-478, Mar. 2013.

[8] K. J. Kim, T. Q. Duong, and X.-N. Tran, "Performance analysis of cognitive spectrum-sharing single-carrier systems with relay selection," IEEE Trans. Signal Process., vol. 60, no. 12, pp. 6435-6449, Dec. 2012.

[9] H. Ding, J. Ge, D. B. d. Costa, and Z. Jiang, "Asymptotic analysis of cooperative diversity systems with relay selection in a spectrum sharing scenario," IEEE Trans. Veh. Technol., vol. 60, no. 2, pp. 457-472, Feb. 2011.

[10] T. Q. Duong, K. J. Kim, H.-J. Zepernick, and C. Tellambura, “Opportunistic relaying for cognitive network with multiple primary users over Nakagami-m fading," in Proc. IEEE ICC 2013, Budapest, Hungary, June 2013, pp. 1-6.

[11] D. S. Michalopoulos and G. K. Karagiannidis, "Distributed switch and stay combining (DSSC) with a single decode and forward relay," IEEE Commun. Lett., vol. 11, no. 5, pp. 408-410, May 2007.

[12] V. N. Q. Bao and H. Y. Kong, "Distributed switch and stay combining for selection relay networks," IEEE Commun. Lett., vol. 13, no. 12, pp. 914-916, Dec. 2009.

[13] M. Yan, Q. Chen, X. Lei, T. Q. Duong, and P. Fan, "Outage probability of switch and stay combining in two-way amplify-and-forward relay networks," IEEE Wireless Commun. Lett., vol. 1, no. 4, pp. 296-299, Jan. 2012.

[14] A. E. Ekpenyong and Y. F. Huang, "Feedback constraints for adaptive transmission," IEEE Signal Process. Mag., vol. 24, no. 3, pp. 69-78, May 2007.

[15] Q. Zhao, S. Geirhofer, L. Tong, and B. Sadler, "Opportunistic spectrum access via periodic channel sensing," IEEE Trans. Signal Process., vol. 56, no. 2, pp. 785-796, Feb. 2008. 